\title{
ELEMENTI (INTER)CULTURALI NEI MANUALI DI ITALIANO LS - LIVELLO A1
}

Gordana Luburić, Università del Montenegro,gbojicic@ucg.ac.me

10.31902/fll.30.2020.19

UDK 37.016:811.131.1(075)

Nel presente lavoro analizzeremo i manuali per l'italiano Ls che vengono utilizzati nell'insegnamento della lingua italiana a livello A1 in Montenegro, concentrando la nostra attenzione in primo luogo sul ruolo della competenza interculturale. Dopo aver presentato il ruolo che essa riveste nel QCER, esamineremo quali sono gli elementi (inter)culturali presentati nei manuali che attualmente vengono utilizzati a diversi livelli di studio in Montenegro, dalle scuole medie e superiori ai corsi universitari. L'obiettivo del lavoro è quello di stabilire in che modo viene integrata la competenza interculturale nei manuali di italiano Ls, quali elementi culturali e in che modo vengono presentati nei manuali nonché di stabilire se la tipologia di elementi culturali e la loro quantità, cambiano a seconda del manuale e dell'età degli apprendenti a cui il manuale è destinato.

Parole chiave: competenza interculturale, elementi culturali, lingua italiana, insegnamento, manuali di italiano Ls

\section{Introduzione}

La comunicazione interculturale, dovuta al processo di globalizzazione e ad una crescente interazione e mobilità intensificata delle persone, diventa un elemento ricorrente nella società plurilinguistica e pluriculturale. Dall'altra parte, le ricerche nel campo della glottodidattica dimostrano che conoscere soltanto gli aspetti formali, ovvero le strutture grammaticali e lessicali di una lingua straniera, fuori dal contesto culturale in cui avviene la comunicazione reale, non permette al discenti di arrivare ad alti livelli di competenza comunicativa. Dato che la lingua, come strumento principale della comunicazione, fa parte della cultura, sarebbe impossibile isolarla dal contesto culturale. Questo perché la comunicazione dipende non solo dalla capacità dell'apprendente di usare una lingua straniera, ma anche dalle sue conoscenze e attitudini sulla propria cultura e su culture di 
altri Paesi, in breve: dalla sua competenza interculturale. Tale competenza aiuta a decodificare varie differenze culturali presenti in un atto comunicativo, nonché a interpretare e produrre messaggi adeguati al contesto comunicativo.

Mitchell and Myles (2004) sottolineano l'interdipendenza della lingua e della cultura, che, secondo gli autori, devono essere acquisite insieme, ognuna delle quali fornisce supporto per lo sviluppo dell'altra. Questa relazione inestricabile della cultura e della lingua viene messa in evidenza anche da Bennett (1997), che introduce il termine fluent $\mathrm{fool}^{1}$, riferendosi alla persona che impara la lingua senza imparare anche la cultura di questa Ls. Questo perché nel processo di apprendimento di una Ls oltre agli aspetti grammaticali e lessicali, un ruolo importante coprono anche gli elementi (inter)culturali.

La competenza interculturale viene vista come una delle competenze chiave da sviluppare in diversi curricula, poiché materie come la storia, la geografia, l'arte, contribuiscono al suo sviluppo. ${ }^{2}$

\footnotetext{
1 "A fluent fool is someone who speaks a foreign language well but doesn't understand the social or philosophical content of the language. [...] Such people [...] may be invited into complicated situations where they cannot understand the events deeply enough to avoid giving or taking offense. Eventually, fluent fools may develop negative opinions of the native speakers whose language they understand but whose basic beliefs and values continue to elude them. To avoid becoming a fluent fool, we need to understand more completely the cultural dimension of language. Language does serve as a tool for communication, but in addition it is a "system of representation" for perception and thinking" (Bennett 16).

2 II Consiglio dell'UE nella Raccomandazione del Consiglio del 22 maggio 2018 relativa alle competenze chiave per l'apprendimento permanente tra le otto competenze chiave, necessarie "per la realizzazione e lo sviluppo personali, la cittadinanza attiva, l'inclusione sociale e l'occupazione" sottolinea l'importanza della comunicazione interculturale e delle abilità interculturali che "sottendono a tutte le competenze chiave". Per quanto riguarda la cultura, la competenza in materia di consapevolezza ed espressione culturali "implica la comprensione e il rispetto di come le idee e i significati vengono espressi creativamente e comunicati in diverse culture e tramite tutta una serie di arti e altre forme culturali. Presuppone l'impegno di capire, sviluppare ed esprimere le proprie idee e il senso della propria funzione o del proprio ruolo nella società in una serie di modi e contesti". Inoltre, nello stesso documento leggiamo che la competenza alfabetica funzionale "implica l'abilità di comunicare e relazionarsi efficacemente con gli altri in modo opportuno e creativo". La competenza multilinguistica come capacità di uso di diverse lingue sottolinea l'importanza di competenza
} 
L'insegnamento delle lingue straniere è di particolare importanza nello sviluppo di questa competenza, che è strettamente legata alla competenza comunicativa, obiettivo dell'insegnamento stesso.

$\mathrm{Nel}$ nostro lavoro cercheremo di stabilire in che modo i manuali di italiano lingua straniera in uso nell'insegnamento dell'italiano in Montenegro aiutino l'apprendente nello sviluppo della competenza interculturale.

\section{Competenza interculturale e competenza comunicativa interculturale}

Per poter analizzare gli elementi culturali presenti nei manuali, il loro rapporto con la competenza comunicativa e il ruolo che la competenza interculturale riveste nei manuali, bisogna prima partire da alcune definizioni della competenza interculturale e della competenza comunicativa interculturale.

II modello più citato è quello proposto da Byram, che sottolinea il bisogno di distinguere queste due competenze. Infatti, secondo l'autore (Byram 1997, 71) la competenza interculturale si riferisce alla capacità delle persone di interagire nella loro lingua con le persone di un altro Paese e cultura, mentre la competenza comunicativa interculturale prende in considerazione l'insegnamento delle lingue e si concentra sulla capacità di interagire con le persone provenienti da un altro Paese e cultura in una lingua straniera.

Una persona che possiede una competenza interculturale è in grado di comprendere e interpretare culture diverse e di agire come mediatore tra esse (Byram, 2008). Pertanto, l'autore definisce parlante interculturale una persona competente in una lingua straniera che possiede la capacità di comprendere le diversità sociali e la realtà di altre culture e di utilizzare la lingua in modo efficace ed appropriato.

Byram descrive il suo modello come una combinazione di conoscenze (savoirs), abilità (savoir faire) e atteggiamenti (savoir-être) che consentono a un parlante di riconoscere, comprendere, interpretare e accettare altri modi di vivere e pensare oltre la propria cultura (Beacco, Byram, 2007).

Guilherme (297) definisce la competenza interculturale come capacità di interagire efficacemente con persone di culture diverse dalla nostra.

interculturale perché "le competenze linguistiche comprendono una dimensione storica e competenze interculturali".

(https://eur-lex.europa.eu/legal-

content/IT/TXT/HTML/?uri=CELEX:32018H0604(possiamo01)\&from=EN) 
Per Vujović (658) la competenza interculturale è la capacità di interpretare in modo adeguato modelli, valori e norme di una cultura straniera e di utilizzarli in un dato contesto.

Fantini (9) definisce la competenza comunicativa interculturale come complesso di abilità necessarie ad agire in modo efficace e appropriato nella comunicazione con gli altri che sono diversi dal punto di vista linguistico e culturale.

Tra le varie competenze all'interno della competenza comunicativa, Coppola (5) annovera anche la competenza culturale, che secondo l'autrice "chiama in causa la scena culturale in cui l'evento ha luogo, e dunque le norme socio-culturali, i valori, le consuetudini, i comportamenti dei partecipanti", e la competenza transculturale e la competenza interculturale. Secondo l'autrice queste due competenze "mettono in luce l'aspetto di variabilità culturale", la competenza transculturale mediante un approccio comparativo (confronto tra culture diverse), mentre la competenza interculturale si basa su un approccio interazionale (incontro tra culture diverse).

Visto che la competenza comunicativa include lo sviluppo di diverse abilità linguistiche, anche la competenza comunicativa interculturale presuppone sviluppo di alcune abilità specifiche. Balboni (7) individua le seguenti abilità: saper osservare, decentrarsi e straniarsi; saper sospendere il giudizio; saper relativizzare; saper ascoltare attivamente; saper comprendere emotivamente e saper negoziare i significati. Anche queste abilità dimostrano l'importanza di allontanarsi dai propri riferimenti valoriali, di non considerare la propria cultura e il proprio modo di pensare come gli unici giusti possibili e di capire il valore di un'altra cultura.

\section{Competenza interculturale nel QCER}

Secondo il QCER (125) la competenza comunicativa comprende le competenze generali e le competenze linguistico-comunicative.

Tra le competenze generali noi citiamo solo le conoscenze dichiarative (sapere), ovvero la conoscenza del mondo, la conoscenza socioculturale $^{3}$ e la consapevolezza interculturale; le abilità e il saper

3 Byram (1997) sottolinea un'importante differenza tra la competenza socioculturale e la competenza interculturale in riferimento all'insegnamento delle Ls. Infatti, se l'obiettivo dell'insegnamento è il raggiungimento della competenza socioculturale, di solito tale insegnamento implica solo la cultura della Ls. Dall'altra parte, se l'insegnamento mira al raggiungimento della competenza interculturale, oltre alla cultura della Ls, sono presenti anche la cultura della L1 ed altre culture. 
fare, ovvero le abilità pratiche, le abilità interculturali ${ }^{4}$ perché parlano del ruolo che la cultura riveste nell'apprendimento di una lingua straniera.

La sezione dedicata alla conoscenza socioculturale (QCER, 127) elenca alcuni elementi che caratterizzano una particolare società e la sua cultura: la vita di tutti i giorni; le condizioni di vita; le relazioni interpersonali; valori, convinzioni e atteggiamenti; il linguaggio del corpo; le convenzioni sociali e i comportamenti rituali.

Nonostante si parli dell'importanza del legame tra lingua e cultura, delle conoscenze socioculturali e della competenza interculturale, nel QCER troviamo solo delle indicazioni piuttosto generiche, mentre mancano una definizione della competenza comunicativa interculturale ed eventuali descrittori per questa competenza.

\section{Come integrare la competenza interculturale nell'insegnamento delle lingue straniere?}

Liddicoat et al. (Liddicoat et al. 2003, 9) sostengono che c'è un'interazione costante tra lingua e cultura e che la cultura si collega a tutti i livelli dell'uso della Ls e delle sue strutture. Per questo, secondo gli autori, non esiste nessun livello della lingua che potrebbe essere indipendente dalla cultura. L'insegnamento (inter)culturale della lingua straniera dovrebbe dunque avvenire già dai livelli elementari. Bisogna partire dal linguaggio molto semplice che illustra le situazioni comunicative reali come saluti o la scelta del pronome adeguato perché anche queste espressioni semplici portano un "carico" culturale molto significativo che deve essere affrontato quando vengono insegnati questi aspetti linguistici. L'assenza di input sulla cultura crea un vuoto culturale che non può essere riempito in seguito, o potrebbe portare gli apprendenti a formulare delle ipotesi basate sulla propria cultura che in seguito si dimostreranno sbagliate (Liddicoat, 2004).

4 “Le abilità interculturali e il saper fare comprendono:

- la capacità di mettere in rapporto la cultura d'origine con quella straniera;

- la sensibilità culturale e la capacità di individuare e usare opportune strategie per entrare in contatto con persone di altre culture;

- la capacità di fungere da intermediario tra la propria cultura e quella straniera e di risolvere efficacemente fraintendimenti interculturali e situazioni conflittuali;

- la capacità di superare modalità di relazione stereotipate". (QCER, 129) 
Per consentire agli apprendenti di diventare competenti dal punto di vista interculturale, Beltrán-Palanques (65) suggerisce un approccio di insegnamento consistente di cinque fasi: fase iniziale, esplorazione e riflessione, osservazione, interazione e feedback.

Nella prima fase gli apprendenti, rispondendo alle domande poste dall'insegnante, dovrebbero definire la cultura e i rapporti interculturali, nonché il ruolo del parlante interculturale nella società. Dopo che la classe avrà discusso sulle proprie scelte, l'insegnante fornirà una definizione esplicita della cultura, della competenza interculturale e del parlante interculturale. In questa fase, l'insegnante riceve importanti informazioni sugli atteggiamenti, sui valori e sul modo di pensare degli apprendenti, nonché sulle loro conoscenze su questo tema.

Nella seconda fase gli apprendenti confrontano la propria cultura con la cultura della lingua oggetto di studio.

Nella terza fase l'insegnante fornisce l'input che mostra le caratteristiche e le rappresentazioni autentiche della cultura della Ls, evitando gli stereotipi. Gli studenti leggono un testo, poi vedono un breve video e alla fine rispondono ad alcune domande poste dall'insegnante. Queste domande mirano alla cultura della Ls, ma anche alla cultura della L1, visto che l'apprendente è invitato a paragonare le manifestazioni di due culture.

La quarta fase ha come obiettivo l'interazione, ovvero la produzione focalizzata a specifici aspetti culturali. Gli apprendenti vengono messi in contatto con i parlanti nativi quali rappresentanti della cultura target (se non dal vivo, allora attraverso le mail o le reti sociali) e così avvengono i primi incontri interculturali. Questa fase è molto importante perché consente di venire in contatto anche con il linguaggio non verbale.

L'ultima fase, realtiva al feedback, consiste del ripasso e dell'analisi di quanto eseguito nelle fasi precedenti. Vengono analizzati tutti i dati raccolti sia dall'insegnante che dai discenti nel corso delle prime quattro fasi. Questa fase di feedback prende in considerazione quattro categorie di competenze e abilità stabilite da Byram (Byram, 1997): conoscenza interculturale, atteggiamenti interculturali (relativizzare la propria prospettiva e valorizzare gli altri), abilità interculturali (abilità interpretative e abilità di interazione, attraverso il confronto e l'interpretazione delle due culture) e il pensiero culturale critico (l'apprendente diventa un mediatore interculturale).

Affinché i loro alunni non diventino fluent fools, Bennett (20) propone agli insegnanti di lingue straniere di utilizzare un approccio basato sul contrasto delle culture che consiste di questi due passi: a) 
informare gli studenti su come la loro lingua madre è collegata ai valori di base, credenze, modelli e schemi di pensiero nella propria cultura; $b$ ) confrontare i modelli di lingua-cultura di origine con quelli della nuova lingua-cultura, soprattutto quei concetti e quelle strutture della Ls che non esistono nella L1.

Gli insegnanti, dunque, dovrebbero aiutare gli studenti a sviluppare la consapevolezza interculturale e fornire attività in cui la cultura, i valori e i comportamenti di un altro paese vengono analizzati e presi in considerazione (Byram 1997).

L'obiettivo dell'insegnamento di una Ls dovrebbe, quindi, essere aiutare gli apprendenti ad acquisire una conoscenza di base della cultura del paese o dei paesi in cui si parla la Ls, nonché mettere in relazione gli elementi della propria cultura agli elementi della cultura della lingua oggetto di studio.

\section{La competenza interculturale nei manuali di lingue straniere}

Come abbiamo visto, per poter contestualizzare bene la lingua, oltre alle strutture linguistiche, gli apprendenti devono acquisire anche i modelli culturali del Paese la cui lingua studiano. Nel contesto di insegnamento delle lingue straniere, dove l'aula resta l'unico contesto in cui si entra in contatto con l'altra lingua, questi modelli culturali vengono proposti o dall'insegnante o dai materiali didattici. Tra questi due, il ruolo dominante ricoprono i manuali di lingua straniera.

Dunnet et al. (in Skopinskaja 2003: 41) dividono i manuali di Ls in due categorie: monodimensionali e bidimensionali. I primi sono focalizzati alla cultura della lingua straniera e lasciano poca opportunità per il confronto tra due culture, a differenza dei manuali bidimensionali che mirano alla comprensione interculturale. Nei manuali monodimensionali l'insegnante ricopre un ruolo importante, perché deve in qualche modo incentivare le attività che mirano allo sviluppo della competenza interculturale.

L'autrice, inoltre, offre la suddivisione in materiali internazionali e locali. I primi sono destinati agli apprendenti di una Ls in diversi contesti e in diversi Paesi, e di solito i loro autori sono parlanti nativi della Ls oggetto dello studio (Skopinskaja, 42). I manuali locali, invece, vengono redatti in base al curriculum ufficiale nazionale e da parte degli autori locali. Se ne deduce una tendenza a insegnare la cultura straniera da una prospettiva locale. 


\section{Elementi (inter)culturali nei manuali di italiano Ls}

Nel nostro lavoro abbiamo analizzato i manuali di italiano Ls che vengono utilizzati in Montenegro in diversi contesti di studio, dalle scuole medie all'università, al livello elementare A1.

Nelle scuole medie, l'italiano si studia come seconda lingua straniera e l'insegnamento dura quattro anni. II manuale destinato a questo livello di studio è il Progetto italiano junior.

Nelle scuole superiori (liceo ed istituti professionali) l'italiano si studia come seconda lingua straniera, e il manuale previsto dal curriculum è il Nuovo progetto italiano, dallo stesso editore, Edilingua. Alla Facoltà di filologia ${ }^{5}$ l'italiano si studia sia come prima che come seconda lingua straniera, e il manuale previsto dal curriculum è il Nuovo contatto.

Si nota subito che tutti i manuali oggetto della nostra ricerca sono internazionali o monodimensionali, dato che non esiste un manuale di lingua italiana scritto apposta per gli apprendenti madrelingua montenegrini.

Il nostro scopo è stabilire quali elementi culturali vengono presentati agli studenti montenegrini. Abbiamo preso in considerazione ogni elemento culturale relativo alla società italiana, nel campo delle scienze, arti, tradizione, usanze ecc.

\section{Risultati dell'analisi}

La nostra analisi ha dimostrato alcune somiglianze tra i manuali analizzati. Tutti e tre optano per un approccio esplicito alla cultura italiana, dato che in tutti i manuali analizzati abbiamo sezioni dedicate alla realtà socioculturale italiana, di solito separate dall'unità didattica in cui vengono presentati gli aspetti formali della lingua.

Molti testi in cui vengono introdotti elementi culturali non hanno nessuna attività introduttiva, per elicitare le conoscenze, per dare la possibilità agli studenti di formulare delle ipotesi. L'approccio scelto dagli autori si basa sulla lettura del testo in base al quale gli studenti devono rispondere alle domande relative al testo letto e alla realtà italiana moderna. Gli autori dei manuali, però, invitano gli studenti a confrontare due culture, a esprimere la propria opinione su alcuni aspetti (inter)culturali poiché troviamo diverse attività/compiti

\footnotetext{
${ }^{5}$ Soltanto gli studenti che studiano l'italiano come prima lingua straniera presso il Dipartimento di Italianistica della Facoltà di Filologia, nel corso degli studi seguono corsi sulla cultura e sulla civiltà italiana, mentre gli altri studenti acquisiscono le nozioni sulla cultura italiana attraverso le lezioni di lingua.
} 
che richiedono un confronto tra alcuni aspetti culturali che riguardano due Paesi.

I principali elementi culturali presenti nei manuali analizzati rientrano nelle seguenti categorie: cucina, geografia italiana, monumenti, personaggi storici, personaggi dello spettacolo, scrittori, musicisti, registi, attori, stilisti, sportivi, presentatori, politici, opere letterarie, canzoni, film ecc.

Per quanto riguarda le usanze e costumi in Italia, troviamo la descrizione del carattere degli italiani, dei loro costumi (divertimento, tempo libero), feste, gesti, temi attuali, mass media, abitudini alimentari, trasporti.

Nel manuale destinato agli adolescenti, Progetto italiano junior 1, gli elementi (inter)culturali vengono presentati nella sezione intitolata Conosciamo l'Italia, che troviamo dopo ogni unità didattica.

In questo manuale abbiamo potuto determinare le seguenti categorie: cucina italiana (spaghetti, pizza, lasagne, prosciutto di Parma, penne, fettuccine, scaloppine ecc.); monumenti e località italiane (Ponte di Rialto, Palazzo Vecchio, Piazza del Duomo, Piazza del Campo, Colosseo, Piazza del Plebiscito ecc.); personaggi famosi italiani (Colombo, Volta, Galileo Galilei, Meucci, Pirandello, Manzoni, Petrarca, Roberto Benigni, Tiziano Ferro, Nek, Valentino Rossi) e personaggi stranieri (Lionel Messi, Jessica Alba, Avril Lavigne, Orlando Bloom ecc.), undici città e due regioni italiane, undici paesi e due città straniere (Parigi, Barcellona); undici nomi stranieri; due squadre di calcio (Inter e Milan), un canale televisivo (Canale 5); un'opera letteraria (Scusa ma ti chiamo amore), un film italiano (La vita è bella).

I temi relativi alle abitudini e alle usanze degli italiani presenti in questo manuale trattano la scuola in Italia, i mezzi di trasporto urbano, le feste più importanti e le abitudini alimentari degli italiani.

Gli autori di questo manuale stimolano le conoscenze (inter)culturali attraverso diverse attività, quali:

Ci sono somiglianze o differenze tra la realtà italiana e quella del vostro Paese? (p. 70);

Da quello che sapete, notate differenze o somiglianze tra la cucina del vostro Paese e quella italiana? ( $p .83)$.

II manuale Nuovissimo progetto italiano $1^{6}$ della stessa casa editrice, come il Progetto italiano junior, opta per lo stesso titolo della sezione dedicata agli elementi (inter)culturali. Nella nostra analisi abbiamo riscontrato le seguenti categorie: cucina italiana (limoncello,

\footnotetext{
${ }^{6}$ Nelle scuole secondarie si usano diverse edizioni di questo manuale, e noi abbiamo scelto di analizzare l'ultima edizione.
} 
parmigiano, torrone, pandoro, panettone ecc.); monumenti e località italiane (Colosseo, Piazza Navona, il balcone di Giulietta, Maranello, Scala ecc.); personaggi famosi (Bocelli, Botticelli, Paolo Sorrentino, Elena Ferrante, Vasco Rossi, Giuseppe Verdi ecc.); città italiane (18 città) e straniere (Chiacago, New York e Malaga); dieci nomi di persona stranieri; due squadre di calcio (Juve e Milan), un canale televisivo (Canale 5); un'opera letteraria (Divina Commedia), feste italiane (Natale, Carnevale, 25 aprile, 2 giugno, Ferragosto ecc.), eventi culturali e sportivi (palio di Siena, Regata Storica di Venezia).

Attraverso diverse attività di lettura e produzione libera, gli apprendenti hanno la possibilità di imparare qualcosa sull'Italia e sulle sue regioni, sui mezzi di trasporto urbano usati dagli italiani, sul bar italiano, sul Natale in Italia, su alcuni aspetti relativi al telefono (numeri utili e prefissi di alcune città italiane).

Soltanto in questo manuale troviamo un proverbio (Natale con i tuoi, Pasqua con chi vuoi, p. 84)

Molto importante risulta il fatto che gli apprendenti sono spesso invitati a confrontare la propria cultura con quella italiana. Così troviamo le seguenti attività:

$\mathrm{Nel}$ vostro paese, cosa fanno le persone nel tempo libero? Le percentuali sono le stesse dell'Italia? (p. 41);

Quali sono i tre numeri di emergenza più importanti nel tuo Paese? (p. 55);

Quali sono le feste più importanti nel vostro Paese? (p. 83).

Dal punto di visto quantitativo, il manuale più ricco di elementi culturali è Nuovo contatto A1. Nel manuale troviamo due sezioni dedicate agli elementi (inter)culturali: Dossier cultura e Confronto tra culture.

I temi culturali scelti dagli autori sono simili ai temi presenti negli altri due manuali. Gli elementi culturali vengono presentati attraverso: la cucina italiana con ben 22 piatti menzionati (ravioli, lasagne, penne, orecchiette, tortellini, zampone, struffoli, babà, cassata siciliana, bonnet ecc.), monumenti e località (Arena di Verona, Musei Capitolini, Ercolano, Galleria degli Uffizi, la Torre degli Asinelli, palazzo dei Normanni ecc.); personaggi famosi (Dario Fo, Oliviero Toscani, Carla Fracci, Gianna Nannini, Bocelli, Garibaldi, Casanova, Pavarotti, Bruneleschi, Giotto ecc.) e due personaggi stranieri (Barack Obama e Nelson Mandela); diciotto città italiane, tutte le regioni italiane e inoltre termini come Pianura Padana, isole Eolie, mar Ionio, mar Tirreno, Stromboli ecc. Per quanto riguarda le città straniere, nell'unità didattica relativa agli etnici, troviamo ben 32 città straniere, nonché sei paesi stranieri. Nella stessa unità didattica vengono 
menzionati 40 nomi di persona stranieri, il che rende questo manuale il più ricco tra tutti i tre analizzati. Un'altra caratteristica molto importante di questo manuale è che già nella prima unità affronta il tema dei gesti, un elemento di linguaggio non verbale, importante per lo sviluppo della competenza interculturale.

In ogni unità didattica abbiamo le attività che, partendo dalle informazioni o dai testi che si riferiscono alla cultura italiana, richiedono il confronto delle due culture:

Confronta gli orari e i giorni di apertura e chiusura in Italia e nel tuo Paese (p. 38);

Com'è un pasto tipico italiano? E nel tuo Paese? (p. 79);

Nel tuo Paese molte persone usano la bicicletta per andare a scuola o al lavoro? Quali sono i mezzi di trasporto usati nella tua città? (p. 99).

Analizzando tre manuali utilizzati nell'insegnamento di lingua italiana in Montenegro abbiamo potuto determinare che gli elementi socio-culturali italiani vengono presentati attraverso la cucina italiana; personaggi storici e personaggi famosi quali scrittori, poeti, sportivi, presentatori, musicisti, registi, film e opere letterarie di autori italiani; caratteristiche geografiche e climatiche; monumenti e località.

Per quanto riguarda lo stile di vita degli italiani, le loro usanze e i costumi, nei manuali abbiamo riscontrato i seguenti argomenti: la scuola italiana, le feste più importanti, il modo in cui gli italiani trascorrono il tempo libero, i mezzi di trasporto urbano, le abitudini alimentari e il bar italiano ecc.

\section{Conclusione}

Poiché le ricerche dimostrano che per il raggiungimento di competenza comunicativa bisogna possedere anche la competenza interculturale, gli apprendenti devono analizzare input con numerosi elementi culturali. Per questo i manuali di Ls hanno un ruolo importante nell'acquisizione di competenza interculturale perché tra tutti i materiali didattici possiedono il maggior numero di elementi culturali.

I manuali che abbiamo analizzato seguono gli interessi degli apprendenti ai quali sono destinati e in base a questi interessi scelgono anche i contenuti culturali. Gli autori dei manuali offrono loro l'opportunità di acquisire una conoscenza di base della cultura del Paese di cui studiano la lingua, nonché diverse occasioni per mettere in relazione elementi della propria cultura ad elementi della cultura italiana. Attraverso diverse attività di produzione libera gli autori dei 
manuali chiedono all'apprendente di confrontare gli aspetti culturali dei due Paesi.

La nostra analisi ha dimostrato che i manuali di italiano Ls optano per sezioni culturali separate dal resto dell'unità didattica. Nonostante i tre manuali siano destinati agli apprendenti di diversa età, gli elementi culturali che in essi vengono presentati appartengono alle stesse categorie: peculiarità geografiche, personaggi famosi, cucina, feste e trasporti. Mancano gli elementi culturali relativi alla comunicazione non verbale, nonché i proverbi, modi di dire e fraseologismi. Questo si spiega col fatto che si tratta di livello iniziale di studio di lingua italiana, per il quale questi elementi non sono previsti neanche dal QCER.

Naturalmente, nessun manuale può comprendere tutti gli aspetti culturali e per questo un ruolo importante hanno anche gli insegnanti che dovrebbero possedere un alto livello di competenza interculturale.

La nostra ricerca ha anche limitazioni, perché abbiamo preso in considerazione soltanto i manuali di livello A1, mentre sarebbe interessante continuare le ricerche analizzando i manuali per altri livelli per poter determinare se la tipologia e la quantità di elementi (inter)culturali e l'approccio alla cultura cambino a seconda del livello di competenza. Sarebbe utile analizzare anche l'opinione degli studenti e degli insegnanti riguardo alla competenza interculturale, agli elementi culturali che, secondo loro, rappresentano meglio la società italiana, per determinare se ci sono eventuali stereotipi e pregiudizi, molto importanti nel campo della competenza interculturale.

\section{Bibliografia :}

Balboni, Paolo Ernesto. "La comunicazione interculturale e l'approccio comunicativo: dall'idea allo strumento." EL.LE Vol. 4 - Num. 1 (2015): 7-8.

Beacco, Jean-Claude e Michael Byram. From linguistic diversity to plurilingual education: Guide for the development of language education policies in Europe. Strasbourg: Council of Europe Publishing. Language Policy Division, 2007.

Beltrán-Palanques, Vincent. "Fostering intercultural communicative competence in the foreign language classroom: pedagogical implications." International Journal of English Language \& Translation Studies, 2(2) (2014): 59-70. 
Bennett, Milton J. "How not to be a fluent fool: Understanding the cultural dimensions of language." New ways in teaching culture. New ways in TESOL series II: Innovative classroom techniques. Ed. Alvino E. Fantini. Alexandria, VA: TESOL, 1997. 16-21.

Bozzone Costa, Rosella, Chiara Ghezzi e Monica Piantoni. Nuovo contatto A1. Corso di lingua e civiltà italiana per stranieri. Torino: Loescher, 2014.

Byram, Michael. Teaching and assessing intercultural communicative competence. Clevedon: Multilingual Matters, 1997.

----------From foreign language education to education for intercultural citizenship. Bristol: Multilingual Matters, 2008.

Coppola, Daria Carmina. "Chi insegna italiano non riceve spari". Prospettiva dialogica e interculturalità nell'insegnamento di lingue e culture altre." Scienza e Pace, (2012): 1-16.

Fantini, Alvino E. "Exploring and assessing intercultural competence (CSD Research Paper No. 07-01)". St. Louis, MO: Washington University, Center for Social Development, 2007.

Guilherme, Maria Manuela. "Intercultural competence". Routledge encyclopedia of language teaching and learning. Ed. Michael Byram. London: Routledge, 2000. 297-300.

Liddicoat, Anthony J. et al. Report on intercultural language learning. Commonwealth of Australia: Canberra ACT, 2003.

Liddicoat, Anthony J. "Intercultural language teaching: Principles for practice". New Zealand Language Teacher, 30 (2004): 17-24.

Marin, Telis e Antonella Albano. Progetto italiano Junior 1. Corso multimediale di italiano per adolescenti. Roma: Edilingua, 2011.

Marin, Telis. Nuovissimo Progetto italiano 1. Corso di lingua e civiltà italiana. A1-A2. Roma: Edilingua, 2019.

Mitchell, Rosamond e Florence Myles. Second language learning theories (2nd ed.), London: Arnold, 2004.

Quadro comune europeo di riferimento per le lingue: apprendimento, insegnamento, valutazione Firenze: La Nuova Italia, 2002.

Skopinskaja, Liljana. "The Role of Culture in Foreign Language Teaching Materials: An Evaluation from an Intercultural Perspective". Incorporating Intercultural Communicative Competence in Language Teacher Education. Ed. Lázár Ildikó. Strasbourg: Council of Europe /ECML, 2003. 39-68.

Vujović, Ana. "Obrazovanje profesora stranih jezika u oblasti strane kulture". Pedagogija, 62(4) (2007): 657-666. 
Sitografia:

Raccomandazione del Consiglio del 22 maggio 2018 relativa alle competenze chiave per l'apprendimento permanente (https://eur-lex.europa.eu/legalcontent/IT/TXT/HTML/?uri=CELEX:32018H0604(possiamo01)\& from=EN)

\section{(INTER)KULTURNI ELEMENTI U UDŽBENICIMA ITALIJANSKOG KAO STRANOG JEZIKA - NIVO A1}

U radu ćemo analizirati udžbenike italijanskog kao stranog jezika koji se koriste u nastavi italijanskog jezika na nivou A1 u Crnoj Gori, sa posebnim osvrtom na interkulturnu kompetenciju. Nakon uvida u ulogu interekulturne kompetencije u Zajedničkom evropskom referentnom okviru za žive jezike, utvrdićemo koji su (inter)kulturni elementi zastupljeni u udžbenicima koji se koriste u različitim kontekstima nastave u Crnoj Gori - od osnovne škole do nastave italijanskog jezika na univerzitetskom nivou. Cilj istraživanja je da utvrdimo na koji način se interkulturna kompetencija integriše u udžbenike italijanskog jezika, koji se elementi italijanske kulture i na koji način prezentuju u udžbenicima, kao i da utvrdimo da li se vrsta i količina kulturoloških elemenata razlikuje u zavisnosti od udžbenika i uzrasta učenika kojima je udžbenik namijenjen.

Ključne riječi: interkulturna kompetencija, elementi kulture, italijanski jezik, nastava, udžbenici za italijanski Ls 